\title{
Computational Study of 3,4-Diphenyl-1,2,5-Thiadiazole 1-Oxide for Organic Photovoltaics
}

\author{
Daniel Glossman-Mitnik \\ Grupo NANOCOSMOS, Centro de Investigación en Materiales Avanzados, SC, Miguel de Cervantes 120, \\ Complejo Industrial Chihuahua, Chihuahua, Chih. 31109, Mexico \\ Correspondence should be addressed to Daniel Glossman-Mitnik, daniel.glossman@cimav.edu.mx
}

Received 26 July 2009; Revised 28 October 2009; Accepted 31 October 2009

Recommended by Mohamed Sabry Abdel-Mottaleb

We make use of a model chemistry within Density Functional Theory (DFT) recently presented, which is called CHIH-DFT, to calculate the molecular structure of 3,4-diphenyl-1,2,5-thiadiazole 1-oxide (TSO), as well to predict its infrared (IR), ultraviolet (UV-Vis) and fluorescence (Fluo) spectra, the dipole moment and polarizability, the free energy of solvation in different solvents as an indication of solubility, and the chemical reactivity parameters that arise from Conceptual DFT. The calculated values are compared with the available experimental data for this molecule as a means of validation of our proposed model chemistry.

Copyright (C) 2009 Daniel Glossman-Mitnik. This is an open access article distributed under the Creative Commons Attribution License, which permits unrestricted use, distribution, and reproduction in any medium, provided the original work is properly cited.

\section{Introduction}

Since the dawn of history, man has been fascinated by the Sun, the provider of the light and warmth that sustains life on Earth. In preindustrial times, our major sources of energy wood, wind and water power derived from solar energy. The subsequent discovery and massive exploitation of fossil fuels laid down in the Earth's crust by early aeons of photosynthetic activity have conditioned the developed world to be dependent on convenient, readily available energy [1].

Despite much effort, semiconductor photovoltaic devices made with traditional inorganic semiconductors have remained sufficiently expensive that their uses are confined to a number of niches. Much effort is currently directed towards the use of thin-film semiconductors, in place of silicon wafers, since the direct fabrication of thin devices on substrates offers the prospect of lower manufacturing costs, particularly for larger area applications. An alternative approach is the use of organic, molecular semiconductors, which can be processed over large areas at relatively low temperatures, either by vacuum sublimation of molecular materials, or, preferably, by processing from solution of film-forming materials such as polymers [2].
The objective of this work is to perform a detailed calculation of the molecular structure of 3,4-diphenyl-1,2,5thiadiazole 1-oxide (TSO), as well as to predict its infrared (IR), ultraviolet (UV-Vis), fluorescence (Fluo) spectra, some physical properties related to solubility and the chemical reactivity, by using a model chemistry within Density Functional Theory (DFT) [3] specially tailored to study heterocyclic systems [4] and to validate the calculated results by comparison with the available experimental data related to the molecular structure for this molecule. This compound has several desirable characteristics related to its use in Organic Photovoltaics and OLEDS: (i) it contains the 1,2,5thiadiazole 1-oxide group, with molecular parameters similar to the thiophene 1-oxide derivatives that have been shown to be useful as nanostructured molecular materials; (ii) the $\pi$-conjugated derivatives are generally efficient fluorophores, and as such, useful for the fabrication of nanobiosensors; (iii) it can be used as an attractive building block for Organic Molecular Materials.

\section{Theory and Computational Details}

All computational studies were performed with the Gaussian $03 \mathrm{~W}[5]$ series of programs with density functional methods 
as implemented in the computational package. The equilibrium geometries of the molecules were determined by means of the gradient technique. The force constants and vibrational frequencies were determined by the FREQ calculations on the stationary points obtained after the optimization to check if there were true minima. Density functionals used in this study are a modification of those incorporated in the Gaussian 03W computational package [5]. To this end, we have defined a new model chemistry that we have found empirically that works well with heterocyclic molecules. The model was found by trial and error determination of the molecular structure of several heterocycles and a comparison with the experimental results. The implementation is a slight different version of the PBE0 hybrid density functional $[6,7]$. In the PBE0 (or PBE1PBE one-parameter) functional, there is only one coefficient which is theoretically adjusted to 0.25 , reflecting the mix of Hartree-Fock or exact exchange and the DFT exchange which is represented by the PBE density functional [8]. The correlation part is also represented by the PBE correlation functional [8] with coefficient equal to one. Our proposed density functional model, which we have called PBEg, is the same as PBE0, but with the mixing coefficient $g$ which adopts different values depending on the number of heteroatoms in the studied molecule, or, in turn, of its molecular structure. The value of $g$ can be calculated through the following empirical formula: $g=$ $0.02+0.14 \times \mathrm{FHA} \times \mathrm{FV}+0.03 \times \mathrm{AHA}$, where $\mathrm{FHA}$ is the first heteroatom chosen as the one less electronegative, $\mathrm{FV}$ is valence factor which represents the oxidation state of the FHA (i.e., 1 for the first oxidation state, two for the second, and so on), and AHA are the number of additional heteroatoms besides the FHA. FHA is always equal to one. This formula has been empirically found by studying a large number of heterocyclic compounds with different number of heteroatoms and oxidation states. For example, for the 1,2,5thiadiazole molecule, the $g$ coefficient will be $g=0.02+$ $0.14 \times 1+0.03 \times 2=0.22$, thus implying that $22 \%$ of $\mathrm{HF}$ exchange will be mixed with $78 \%$ of PBE exchange. Similarly, for 1,2,5-thiadiazole 1,1-dioxide, the $g$ coefficient will be $g=$ $0.02+0.14 \times 3+0.03 \times 2=0.50$, reflecting $50 \%$ of HF exchange and $50 \%$ of PBE exchange. For those cases in which the molecule has several heterocyclic rings, we will calculate the coefficient $g$ by averaging the coefficient for each heterocycle. As it is evident, the bounds to the empirical formula are 0 and 1 meaning no mixing of HF exchange in the first case and a purely HF model in the last case. The reasons for the dependence of the mix of HF and DFT exchange with the number of heteroatoms and their oxidation state are not apparent, but the validity of the empirical formula have been computationally assessed for several different molecular systems $[4,9-20]$. Based on these results it is clear that our choice of this particular functional has been done because its performance is superior to other methods both in accuracy and computational cost.

A model chemistry is an unbiased, uniquely defined, and uniformly applicable theoretical model for predicting the properties of chemical systems. A model chemistry generally consists of the combination of a theoretical method wit a basis set [21-30]. In order to define our model chemistry, we have to couple the proposed density functional with one or more basis sets. In this way, the new model chemistry that we have called CHIH-DFT can be represented by the expression $\mathrm{CHIH}=\mathrm{PBEg} /$ basis sets. There are three different CHIH-DFT model chemistries: $\mathrm{CHIH}$ (small) that uses the $3-21 \mathrm{G}^{*}$ basis set for geometry optimizations and frequency calculations, and the CBSB2** basis set for the calculation of the electronic properties; $\mathrm{CHIH}$ (medium) that uses the CBSB2** basis set for geometry optimizations and frequency calculations and the CBSB4 basis set for the electronic properties; and $\mathrm{CHIH}$ (large) which uses the CBSB7 basis set for geometry optimizations and frequency calculations and the CBSB1 basis set for the electronic properties. In this way, by considering a compromise between accuracy and CPU time, the $\mathrm{CHIH}$ (large) will be used for small heterocyclic molecules, the $\mathrm{CHIH}$ (medium) for mediumsized molecules and the $\mathrm{CHIH}$ (small) for large heterocyclic molecules. We understand that a molecule is small when the number of atoms within the cycle is five or six. When the number of atoms in the cycle is more than six, we considered it a medium-sized molecule. If there is more than one cycle, or if there are a large number of sidechain radical substituents, we say that the molecule is large. The CHIH-DFT model chemistry has been already validated for the case of antiparasitic drugs [9-11], flavonoids [1214], organic corrosion inhibitors [15-17], alkaloids [18, 19] and antitubercular drugs [20]. Thus, in our case for the 3,4-diphenyl-1,2,5-thiadiazole 1-oxide molecule (TSO), the value of the mixing will be $g=0.36$, implying that $36 \%$ of Hartree-Fock (HF) exchange is mixed with $64 \%$ of DFT exchange. The correlation part is taken as the PBE correlation functional with coefficient equal to one [8].

For the purpose of comparison, the molecular structure of the molecule has been computed using different model chemistries. Additional density functionals used in this study, are LSDA or SVWN [31, 32], BLYP [33, 34], B3LYP [34, 35], B3PW91 [35, 36], PBEPBE [8], PBE0 [7], TPSSTPSS [37] and TPSSh [38]. A suitable description of these density functionals is provided in some of the most important Computational Chemistry recent books $[25,27,39,40]$.

The calculation of the ultraviolet (UV-Vis) and fluorescence (Fluo) spectra of 3,4-diphenyl-1,2,5-thiadiazole 1-oxide (TSO) has been performed by solving the time dependent Kohn-Sham equations according to the method implemented in Gaussian 03W [39, 41-43]. The equations have been solved for 10 excited states.

The infrared (IR), ultraviolet (UV-Vis), and fluorescence (Fluo) spectra were calculated and visualized using the Swizard program [44]. In all cases the displayed spectra show the calculated frequencies and absorption or emission wavelengths.

\section{Results and Discussion}

The results for the equilibrium conformation of the neutral molecule of 3,4-diphenyl-1,2,5-thiadiazole 1-oxide (or TSO, for short) calculated with the $\mathrm{CHIH}$ (small) model chemistry are presented in Figure 1 through a representation 


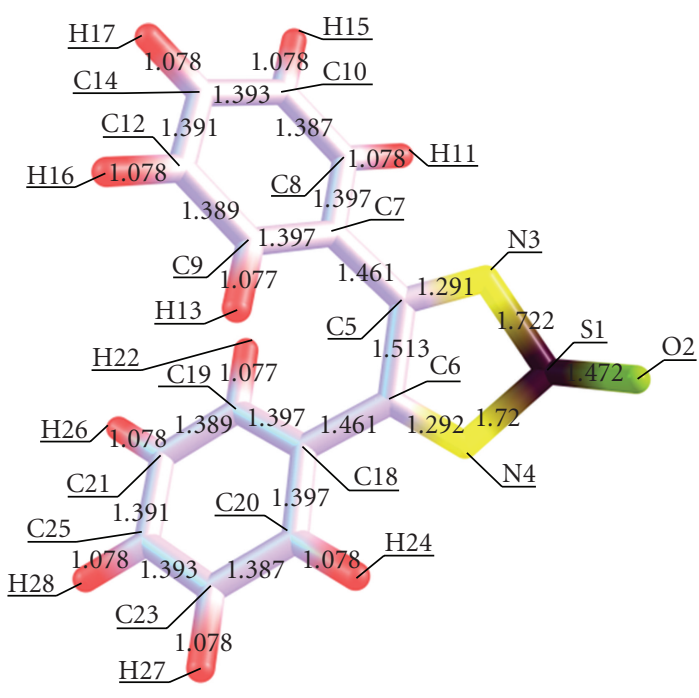

(a)

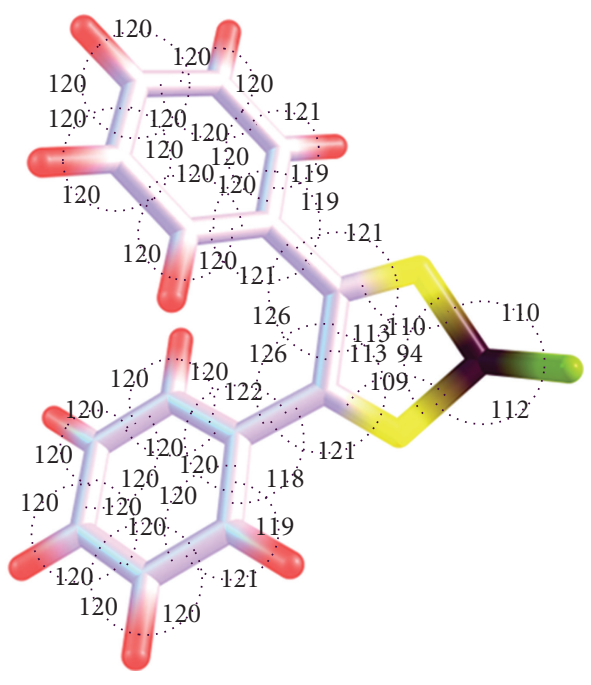

(b)

Figure 1: Interatomic bond distances $(\AA)$ and bond angles (deg) for 3,4-diphenyl-1,2,5-thiadiazole 1-oxide.

of the molecular structure showing the atomic labelling and numbering as well as the interatomic bond lengths and angles. The results have been compared with the experimental X-ray crystallography determination of the molecular structure of TSO [45]. The agreement is generally good: the standard error of the differences between the experimental and the calculated bond lengths and bond angles being very low. Although not shown in Figure 1, an analysis of the torsional angles reveals that the heterocycle is almost planar. The good agreement with the experimental data is more noticeable for the heterocyclic ring, because the model have been parameterized for this kind of systems [4].

As an additional comparison, we have calculated the optimized structure of the studied molecule, using several different common density functionals (local, nonlocal GGA, hybrid, meta-GGA and meta-GGA hybrid) coupled with the CBSB2** basis set. The molecular structure obtained from each calculation was compared with the experimental results by aligning both structures and calculating the Root MeanSquare Deviation (RMSD). The results can be summarized as follows: $\mathrm{CHIH}($ small $)=0.026, \mathrm{PBE} 0=0.033$, TPSSh $=0.049, \mathrm{~B} 3 \mathrm{LYP}=0.054, \mathrm{LSDA}=0.066$, TPSS $=0.053$, $\mathrm{PBEPBE}=0.053, \mathrm{BLYP}=0.087$. It can be concluded that the $\mathrm{CHIH}$ (small) model chemistry performs better than other hybrids functionals and even better than a recently proposed hybrid meta-GGA functional. The behavior of CHIH(small) and is similar and better than local and nonhybrids functionals. This is remarkable if we consider that this model chemistry is more efficient from a computational point of view.

The molecular dipole moment is perhaps the simplest experimental measure of charge distribution in a molecule. The accuracy of the overall distribution of electrons in a molecule is hard to quantify, since it involves all the multipoles. From the present calculations, the total energy, the total dipole moment and the isotropic polarizability of the ground state with the $\mathrm{CHIH}$ (small) model chemistry are $-1121.415 \mathrm{au}, 6.9576$ Debye, and 66.94 $\mathrm{Bohr}^{3}$ for the TSO molecule. These results could be of interest as an indication of the solubility and chemical reactivity of the studied molecule, not only for it synthesis but for the potential polymerization as a step in its use in organic electronics and photovoltaics.

The infrared spectrum (IR) for the TSO molecule calculated with the $\mathrm{CHIH}$ (small) model chemistry is displayed in Figure 2. To the best of our knowledge, the experimental spectrum has not been reported in the literature. The vibrational band assignments have been done using the ChemCraft for Windows molecular visualization program. The symmetric vibration of the SO group has been found at $1225 \mathrm{~cm}^{-1}$. The symmetric stretching of the $\mathrm{C}=\mathrm{N}$ double bonds is located at $1595 \mathrm{~cm}^{-1}$. At $1319 \mathrm{~cm}^{-1}$ there is a signal belonging to heterocyclic C-C symmetric stretching, while at $752 \mathrm{~cm}^{-1}$ there is a strong peak that corresponds to the vibrations of all the aromatic ring hydrogens. These are by far the most intense signals in the IR spectrum. There are two signals at 549 and $599 \mathrm{~cm}^{-1}$ that belong to the asymmetric and symmetric stretching of the S-N bonds. At $835 \mathrm{~cm}^{-1}$ there is a signal related to the vibrations of only three of the hydrogens of the benzenic rings. The asymmetric and symmetric out-of-plane vibrations of the aromatic ring carbons can be observed at 1070 and $1136 \mathrm{~cm}^{-1}$, respectively. The peak at $999 \mathrm{~cm}^{-1}$ belongs to the vibrations of the C-N-S of the thiadiazole moiety. Finally, the symmetric and asymmetric in-plane vibrations of the aromatic ring carbons are observed at 1549 and $1675 \mathrm{~cm}^{-1}$.

As the experimental IR spectrum has not been reported, the scaling factor could not be calculated. However, from our experience based on previous calculations with similar molecules [4], it is possible to say that the scaling factor 


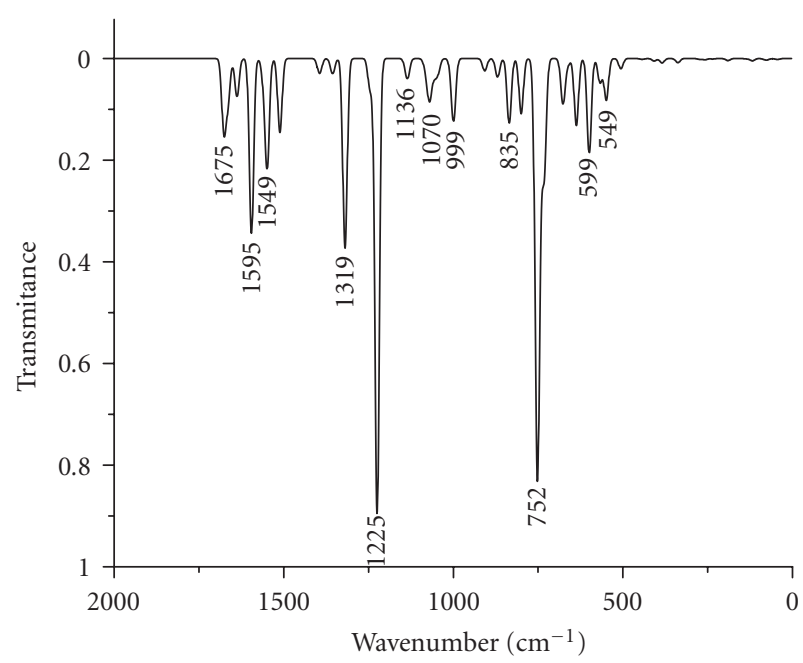

FIGURE 2: Infrared spectrum (IR) of the TSO molecule calculated with the CHIH-DFT(small) model chemistry.

will be between 0.97 and 1 . Thus, it is be expected that the model chemistry used in this work could reproduce the experimental spectrum of the TSO molecule with a certain degree of accuracy.

The ultraviolet spectrum (UV-Vis) of the TSO molecule calculated with the $\mathrm{CHIH}$ (small) model chemistry is displayed in Figure 3. The results for the energy of the excitations and the oscillator strengths computed for the ten low-lying singlet excited states are presented in Table 1. In order to characterize the excited state transitions presented in Table 1, we performed an analysis of all the molecular orbitals involved, taking into consideration that orbital 66 is the HOMO and orbital 67 is the LUMO. For example, in alpha molecular orbital 66 (occupied, $E=-0.038439$ ) main atomic orbitals are: N22 (Px on atom O2) [0.462], N26 (Px on atom O2) [0.429]. In alpha molecular orbital 67 (unoccupied, $E=0.035442$ ) main atomic orbitals are: $\mathrm{N} 24(\mathrm{Pz}$ on atom O2) [0.439], N28 (Pz on atom O2) [0.438]. This means that the HOMO has a $\pi$ character, while the LUMO has a $\pi^{*}$ character. On the basis of the above analysis of transitions and MOs, it can be said that the absorption bands of TSO in the visible and UV regions are typical $\pi-\pi^{*}$ transitions.

As the HOMO-LUMO transition takes place in the ultraviolet region, close to but out of the visible zone, it can be predicted that this molecule will be colorless or slightly colored. In order to calculate the fluorescence spectrum of the studied molecule, the first singlet excited state was optimized by using the CIS/3-21G(d) model chemistry. The results of a TDDFT calculation at the $\mathrm{CHIH}$ (small) model chemistry on the optimized singlet excited state are not shown but reported as the fluorescent spectrum, where the HOMO-LUMO transition takes place at $613 \mathrm{~nm}$, being this signal very weak. This means that the studied molecule will present fluorescence in the visible region. Thus, it could be of potential application for the fabrication of OLEDs.

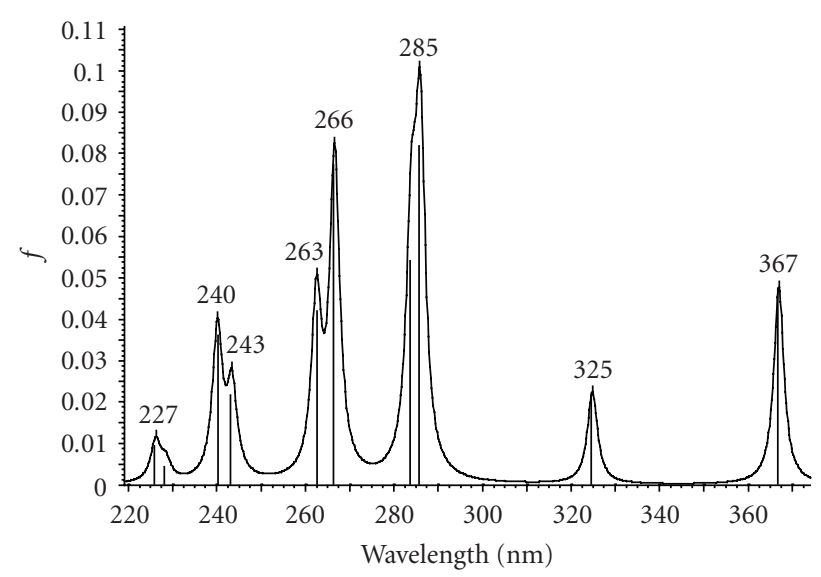

FIgURE 3: UV-Vis spectrum of the TSO molecule calculated with the CHIH-DFT(small) model chemistry.

The free energy of solvation $\Delta G($ solv) of the molecule have been calculated for TSO by resorting to the $\mathrm{CHIH}$ (small) model chemistry coupled with the IntegralEquation-Formalism of the Polarized Continuum Model (IEF-PCM) for different solvents as implemented in Gaussian 03. The solubility of a molecule will depend on several kinetic and thermodynamic factors. However, it can be said that the magnitude and the sign of $\Delta G$ (solv) could be a good approximation as an index of solubility. In this way, a negative sign and a large magnitude will be an indication of increased solubility. The results of these calculations for the studied molecule can be summarized as follows: Chloroform $=-4.56 \mathrm{Kcal} / \mathrm{mol}$, Cyclohexane $=-2.75 \mathrm{kcal} / \mathrm{mol}$, Ethanol $=-10.30 \mathrm{Kcal} / \mathrm{mol}, \mathrm{THF}=-5.18 \mathrm{Kcal} / \mathrm{mol}$, Toluene $=$ $-2.33 \mathrm{Kcal} / \mathrm{mol}$, and Water $=-5.20 \mathrm{Kcal} / \mathrm{mol}$. These values could be an indication that the studied molecule will be mostly soluble in ethanol, and this can be related to the results obtained for the dipole moment and polarizability.

The HOMO and LUMO of TSO calculated with the $\mathrm{CHIH}$ (small) model chemistry are displayed in Figure 4. The HOMO density corresponds to the nitrogens, the oxygen and some carbon atoms of the nonheterocyclic rings, while the LUMO density is over the whole molecule. This can give us an idea of the reactivity of the molecule.

The reactive sites can be identified through an analysis of the total and orbital densities. The representation of the calculated HOMO and LUMO densities in Figure 4 show that the electrophilic attack would occur preferentially at the nitrogens and the nucleophilic attack at the heterocyclic ring carbons.

The condensed Fukui functions can also be employed to determine the reactivity of each atom in the molecule. The corresponding condensed functions are given by $f_{k}^{+}=$ $q_{k}(N+1)-q_{k}(N)$ (for nucleophilic attack), $f_{k}^{-}=q_{k}(N)-$ $q_{k}(N-1)$ (for electrophilic attack), and $f_{k}^{0}=\left[q_{k}(N+1)-\right.$ $\left.q_{k}(N-1)\right] / 2$ (for radical attack), where $q_{k}$ is the gross charge of atom $k$ in the molecule.

It is possible to evaluate condensed Fukui functions from single-points calculations directly, without resorting to 
TABLE 1: Excitation energies and oscillator strengths of 3,4-diphenyl-1,2,5-thiadiazole 1-oxide calculated by using TDDFT and the $\mathrm{CHIH}$ (small) model chemistry.

\begin{tabular}{|c|c|c|c|c|c|}
\hline $\begin{array}{l}\text { Excited State } \\
63 \rightarrow 67 \\
65 \rightarrow 67 \\
66 \rightarrow 67\end{array}$ & 1: & $\begin{array}{c}\text { Singlet-A } \\
0.22354 \\
0.14980 \\
0.60774\end{array}$ & $3.3786 \mathrm{eV}$ & $366.97 \mathrm{~nm}$ & $f=0.0477$ \\
\hline Excited State & 2: & Singlet-A & $3.8166 \mathrm{eV}$ & $324.85 \mathrm{~nm}$ & $f=0.0222$ \\
\hline $61 \rightarrow 67$ & & 0.41771 & & & \\
\hline $63 \rightarrow 67$ & & 0.22445 & & & \\
\hline $64 \rightarrow 67$ & & 0.17251 & & & \\
\hline $65 \rightarrow 67$ & & 0.41396 & & & \\
\hline $66 \rightarrow 67$ & & -0.11265 & & & \\
\hline Excited State & 3: & Singlet-A & $4.3360 \mathrm{eV}$ & $285.94 \mathrm{~nm}$ & $f=0.0819$ \\
\hline $63 \rightarrow 67$ & & -0.31629 & & & \\
\hline $64 \rightarrow 67$ & & 0.56550 & & & \\
\hline $66 \rightarrow 67$ & & 0.19535 & & & \\
\hline Excited State & 4: & Singlet-A & $4.3668 \mathrm{eV}$ & $283.93 \mathrm{~nm}$ & $f=0.0543$ \\
\hline $61 \rightarrow 67$ & & -0.12090 & & & \\
\hline $63 \rightarrow 67$ & & -0.40626 & & & \\
\hline $64 \rightarrow 67$ & & -0.24884 & & & \\
\hline $65 \rightarrow 67$ & & 0.45779 & & & \\
\hline Excited State & 5: & Singlet-A & $4.6504 \mathrm{eV}$ & $266.61 \mathrm{~nm}$ & $f=0.0774$ \\
\hline $60 \rightarrow 67$ & & 0.11497 & & & \\
\hline $61 \rightarrow 67$ & & 0.34959 & & & \\
\hline $62 \rightarrow 67$ & & 0.40642 & & & \\
\hline $63 \rightarrow 67$ & & -0.23124 & & & \\
\hline $64 \rightarrow 67$ & & -0.18987 & & & \\
\hline $65 \rightarrow 67$ & & -0.20023 & & & \\
\hline $66 \rightarrow 67$ & & 0.14139 & & & \\
\hline Excited State & $6:$ & Singlet-A & $4.7230 \mathrm{eV}$ & $262.51 \mathrm{~nm}$ & $f=0.0422$ \\
\hline $61 \rightarrow 67$ & & -0.31143 & & & \\
\hline $62 \rightarrow 67$ & & 0.52106 & & & \\
\hline $63 \rightarrow 67$ & & 0.19105 & & & \\
\hline $64 \rightarrow 67$ & & 0.11226 & & & \\
\hline $64 \rightarrow 68$ & & -0.10636 & & & \\
\hline Excited State & $7:$ & Singlet-A & $5.0935 \mathrm{eV}$ & $243.41 \mathrm{~nm}$ & $f=0.0219$ \\
\hline $58 \rightarrow 67$ & & 0.10081 & & & \\
\hline $59 \rightarrow 67$ & & 0.17992 & & & \\
\hline $60 \rightarrow 67$ & & 0.39836 & & & \\
\hline $61 \rightarrow 67$ & & -0.15856 & & & \\
\hline $66 \rightarrow 68$ & & 0.45570 & & & \\
\hline Excited State & 8: & Singlet-A & $5.1635 \mathrm{eV}$ & $240.12 \mathrm{~nm}$ & $f=0.0363$ \\
\hline $58 \rightarrow 67$ & & 0.12525 & & & \\
\hline $60 \rightarrow 67$ & & 0.47276 & & & \\
\hline $66 \rightarrow 68$ & & -0.44998 & & & \\
\hline Excited State & 9: & Singlet-A & $5.4269 \mathrm{eV}$ & $228.46 \mathrm{~nm}$ & $f=0.0046$ \\
\hline $59 \rightarrow 67$ & & 0.57372 & & & \\
\hline $61 \rightarrow 67$ & & 0.14968 & & & \\
\hline $64 \rightarrow 68$ & & 0.10981 & & & \\
\hline $66 \rightarrow 68$ & & -0.18025 & & & \\
\hline Excited State & 10: & Singlet-A & $5.4817 \mathrm{eV}$ & $226.18 \mathrm{~nm}$ & $f=0.0097$ \\
\hline $59 \rightarrow 67$ & & -0.12433 & & & \\
\hline $62 \rightarrow 67$ & & 0.19734 & & & \\
\hline $62 \rightarrow 71$ & & 0.12404 & & & \\
\hline $63 \rightarrow 68$ & & 0.14583 & & & \\
\hline $63 \rightarrow 69$ & & -0.16390 & & & \\
\hline $63 \rightarrow 70$ & & -0.11249 & & & \\
\hline $64 \rightarrow 68$ & & 0.49784 & & & \\
\hline $65 \rightarrow 68$ & & -0.14970 & & & \\
\hline $65 \rightarrow 70$ & & 0.11729 & & & \\
\hline $66 \rightarrow 69$ & & 0.25687 & & & \\
\hline
\end{tabular}




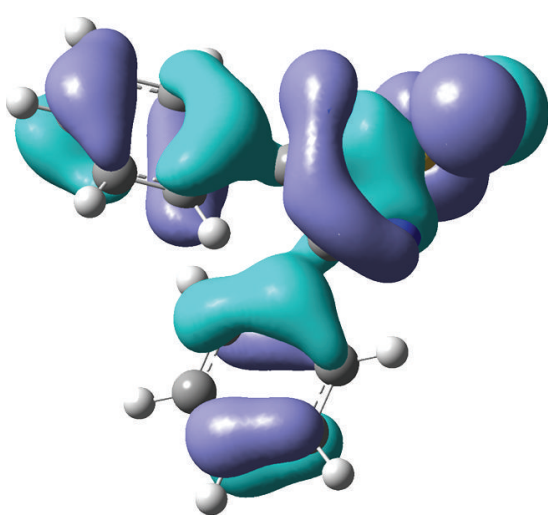

(a)

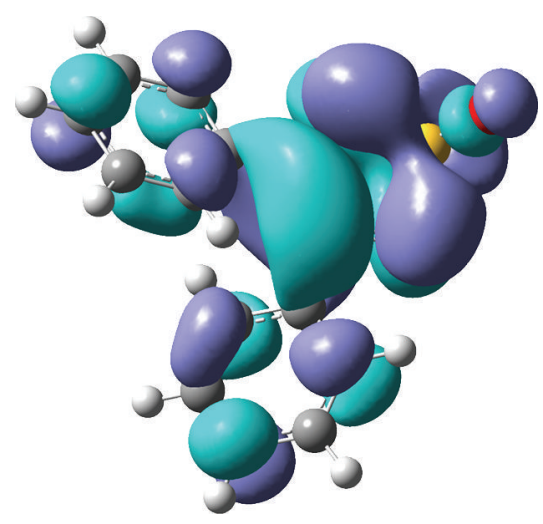

(b)

FIGURE 4: HOMO and LUMO of the TSO molecule calculated with the CHIH-DFT model chemistry.

additional calculations involving the systems with $N-1$ and $N+1$ electrons:

$$
\begin{aligned}
& f_{k}^{+}=\sum_{a \in k}\left[c_{a i}^{2}+c_{a i} \sum_{b \neq a} c_{b i} S_{a b}\right] \quad(\text { where } i=\text { LUMO }), \\
& f_{k}^{-}=\sum_{a \in k}\left[c_{a i}^{2}+c_{a i} \sum_{b \neq a} c_{b i} S_{a b}\right] \quad(\text { where } i=\text { HOMO) }
\end{aligned}
$$

with $c_{a i}$ being the LCAO coefficients and $S_{a b}$ the overlap matrix. The condensed Fukui functions are normalized, thus $\sum_{k} f_{k}=1$ and $f_{k}^{0}=\left[f_{k}^{+}+f_{k}^{-}\right] / 2$.

The results from the calculation of the condensed Fukui functions for nucleophilic, electrophilic, and radical attack have been obtained by resorting to the AOMix molecular analysis program [46] and are presented in Table 2.

The sites for electrophilic attack will be those atoms bearing a negative charge and where the Fukui function $f_{k}^{-}$ is a maximum. These values confirm that the site for the electrophilic attack is the oxygen atom. The site for potential nucleophilic attack would depend on the values of $f_{k}^{+}$on the atoms with a positive charge density. The results form Table 1 show that the site for nucleophilic attack will be the nitrogen atoms and also the heterocyclic carbon atoms. Finally, the sites for radical attack, governed by the values of $f_{k}^{0}$ will be the oxygen atom, and perhaps the sulfur atom. The benzenic rings are not very reactive in this context.

\section{Conclusions}

In this work, our model chemistry within DFT (the CHIHDFT chemistry model) has been presented and the methodology has been applied to the study of a molecule which is potentially useful for OLEDs and photovoltaic devices. The molecular structure for TSO has been determined by using the $\mathrm{CHIH}$ (small) model chemistry. A comparison has been made with the results from the experimental X-ray crystallography for this molecule. The agreement is generally very good. It is worth noting that the results obtained with the (faster) $\mathrm{CHIH}$ (small) model chemistry are very
TABLE 2: Condensed Fukui functions calculated by using the $\mathrm{CHIH}$ (small) model chemistry and through the AOMIX molecular

\begin{tabular}{|c|c|c|c|}
\hline Atom & $f_{k}^{+}$ & $f_{k}^{-}$ & $f_{k}^{0}$ \\
\hline $1 \mathrm{~S}$ & 0.0099 & 0.2253 & 0.1176 \\
\hline $2 \mathrm{O}$ & 0.0022 & 0.2999 & 0.1511 \\
\hline $3 \mathrm{~N}$ & 0.1758 & 0.0071 & 0.0915 \\
\hline $4 \mathrm{~N}$ & 0.1794 & 0.0025 & 0.0910 \\
\hline $5 \mathrm{C}$ & 0.1486 & 0.0023 & 0.0755 \\
\hline $6 \mathrm{C}$ & 0.1533 & 0.0028 & 0.0781 \\
\hline $7 \mathrm{C}$ & 0.0009 & 0.0070 & 0.0039 \\
\hline $8 \mathrm{C}$ & 0.0028 & 0.0018 & 0.0023 \\
\hline $9 \mathrm{C}$ & 0.0026 & 0.0028 & 0.0027 \\
\hline $10 \mathrm{C}$ & 0.0012 & 0.0155 & 0.0083 \\
\hline $11 \mathrm{H}$ & 0.0003 & 0.0001 & 0.0002 \\
\hline $12 \mathrm{C}$ & 0.0046 & 0.0079 & 0.0062 \\
\hline $13 \mathrm{H}$ & 0.0006 & 0.0006 & 0.0006 \\
\hline $14 \mathrm{C}$ & 0.0318 & 0.0572 & 0.0445 \\
\hline $15 \mathrm{H}$ & 0.0001 & 0.0003 & 0.0002 \\
\hline $16 \mathrm{H}$ & 0.0002 & 0.0001 & 0.0002 \\
\hline $17 \mathrm{H}$ & 0.0003 & 0.0004 & 0.0004 \\
\hline $18 \mathrm{C}$ & 0.0011 & 0.0042 & 0.0026 \\
\hline $19 \mathrm{C}$ & 0.0295 & 0.0269 & 0.0282 \\
\hline $20 \mathrm{C}$ & 0.0333 & 0.0057 & 0.0195 \\
\hline $21 \mathrm{C}$ & 0.0045 & 0.0015 & 0.0030 \\
\hline $22 \mathrm{H}$ & 0.0008 & 0.0003 & 0.0005 \\
\hline $23 \mathrm{C}$ & 0.0016 & 0.0177 & 0.0096 \\
\hline $24 \mathrm{H}$ & 0.0004 & 0.0001 & 0.0003 \\
\hline $25 \mathrm{C}$ & 0.0387 & 0.0345 & 0.0361 \\
\hline $26 \mathrm{H}$ & 0.0001 & 0.0004 & 0.0002 \\
\hline $27 \mathrm{H}$ & 0.0001 & 0.0001 & 0.0001 \\
\hline $28 \mathrm{H}$ & 0.0004 & 0.0002 & 0.0003 \\
\hline
\end{tabular}
analysis program.

similar to those obtained using the PBE0 functional with a CBSB7 basis set, thus implying that equally accurate results could be obtained in a reasonable time in the study of 
heterocycles. A general comparison with the results from the optimization of the molecular structure using several different density functionals have also been performed and the conclusion is that the CHIH-DFT is a good alternative to other model chemistries based on hybrids GGA and metaGGA functionals for the prediction of molecular structures.

The shape of the frontier orbitals of this molecule were displayed as well as some electronic parameters like the total energy, the dipole moment and the polarizability. Moreover, the calculation of the condensed Fukui functions can be useful to obtain an idea of the chemical reactivity of the studied molecule. This is of special importance if we consider that the studied molecule could be the starting point for a polymeric material of potential application in organic electronics and photovoltaics, and that the chemical reactivity indices could assist the experimentalists in planing and guiding the synthetic and polymerization processes.

The infrared (IR) and ultraviolet (UV-Vis) and fluorescence (Fluo) spectra for TSO have been predicted according to the $\mathrm{CHIH}$ (small) model chemistry, and an assignment of the principal peaks have been achieved. The shape of the UV-Vis spectra and the maximum absorption wavelength belonging to this molecule have been presented and this have been compared with the fluorescence spectra. The results showed that the studied molecule could be an useful starting point for the preparation of materials of use in organic electronic and photovoltaics.

The free energy of solvation $\Delta \mathrm{G}(\mathrm{solv})$ of the molecule have been calculated for TSO by resorting to the $\mathrm{CHIH}$ (small) model chemistry coupled with the IntegralEquation-Formalism of the Polarized Continuum Model (IEF-PCM) for different solvents and the results gave an indication of ethanol as the solvent in which this molecule could be potentially soluble.

The CHIH-DFT model chemistry appears to be a useful tool for the study of the molecular structure and electronic properties of heterocycles, and further applications to several molecular systems are being pursued in our laboratory.

\section{Acknowledgments}

This work has been partially supported by Consejo Nacional de Ciencia y Tecnologa (CONACYT, Mexico) and by Fondo Mixto del Estado de Baja California (FOMIX-BC) through Project 69363. The author is a researcher of CONACYT and CIMAV.

\section{References}

[1] M. D. Archer and R. Hill, Clean Electricity from Photovoltaics, Imperial College Press, London, UK, 2001.

[2] J. J. M. Halls and R. H. Friend, "Organic photovoltaic devices," in Clean Electricity from Photovoltaics, M. D. Archer and R. Hill, Eds., Imperial College Press, London, UK, 2001.

[3] R. G. Parr and W. Yang, Density Functional Theory of Atoms and Molecules, Oxford University Press, New York, NY, USA, 1989.

[4] D. Glossman-Mitnik, "CHIH-DFT determination of the molecular structure and infrared and ultraviolet spectra of azathiophenes," Theoretical Chemistry Accounts, vol. 117, no. 1, pp. 57-68, 2007.

[5] M. J. Frisch, G. W. Trucks, H. B. Schlegel, et al., Gaussian 03, Revision E.01, Gaussian, Inc., Wallingford, Conn, USA, 2004.

[6] M. Ernzerhof and G. E. Scuseria, "Assessment of the PerdewBurke-Ernzerhof exchange-correlation functional," Journal of Chemical Physics, vol. 110, no. 11, pp. 5029-5036, 1999.

[7] C. Adamo, "Toward reliable density functional methods without adjustable parameters: the PBE0 model," Journal of Chemical Physics, vol. 110, no. 13, pp. 6158-6170, 1999.

[8] J. P. Perdew, K. Burke, and M. Ernzerhof, "Generalized gradient approximation made simple," Physical Review Letters, vol. 77, no. 18, pp. 3865-3868, 1996.

[9] N. Flores-Holguín and D. Glossman-Mitnik, "CHIH-DFT determination of the molecular structure, infrared and ultraviolet spectra of the antiparasitic drug megazol," Journal of Molecular Structure: THEOCHEM, vol. 681, no. 1-3, pp. 7782, 2004.

[10] N. Flores-Holguín and D. Glossman-Mitnik, "CHIH-DFT determination of the electrical, optical, and magnetic properties and NICS aromaticity of megazol," Journal of Molecular Structure: THEOCHEM, vol. 717, no. 1-3, pp. 1-3, 2005.

[11] N. Flores-Holguín and D. Glossman-Mitnik, "CHIH-DFT determination of the reactivity sites of the antiparasitic drug megazol," Journal of Molecular Structure: THEOCHEM, vol. 723, no. 1-3, pp. 231-234, 2005.

[12] A. M. Mendoza-Wilson and D. Glossman-Mitnik, "CHIHDFT determination of the molecular structure, infrared and ultraviolet spectra of the flavonoid quercetin," Journal of Molecular Structure: THEOCHEM, vol. 681, no. 1-3, pp. 7176, 2004.

[13] A. M. Mendoza-Wilson and D. Glossman-Mitnik, "CHIHDFT study of the electronic properties and chemical reactivity of quercetin," Journal of Molecular Structure: THEOCHEM, vol. 716, no. 1-3, pp. 67-72, 2005.

[14] A.M. Mendoza-Wilson and D. Glossman-Mitnik, "Theoretical study of the molecular properties and chemical reactivity of $(+)$-catechin and $(-)$-epicatechin related to their antioxidant ability," Journal of Molecular Structure: THEOCHEM, vol. 761, no. 1-3, pp. 97-106, 2006.

[15] L. M. Rodríguez-Valdez, A. Martínez-Villafañe, and D. Glossman-Mitnik, "CHIH-DFT determination of the molecular structure, infrared and ultraviolet spectra of potentially organic corrosion inhibitors," Journal of Molecular Structure: THEOCHEM, vol. 681, no. 1-3, pp. 83-88, 2004.

[16] L. M. Rodríguez-Valdez, A. Martínez-Villafañe, and D. Glossman-Mitnik, "CHIH-DFT theoretical study of isomeric thiatriazoles and their potential activity as corrosion inhibitors," Journal of Molecular Structure: THEOCHEM, vol. 716, no. 1-3, pp. 61-65, 2005.

[17] L. M. Rodríguez-Valdez, W. Villamisar, M. Casales, et al., "Computational simulations of the molecular structure and corrosion properties of amidoethyl, aminoethyl and hydroxyethyl imidazolines inhibitors," Corrosion Science, vol. 48, no. 12, pp. 4053-4064, 2006.

[18] D. Glossman-Mitnik, "CHIH-DFT determination of the molecular structure and IR and UV spectra of solanidine," Journal of Molecular Modeling, vol. 13, no. 1, pp. 43-46, 2007.

[19] D. Glossman-Mitnik, "CHIH-DFT determination of the molecular structure and infrared and ultraviolet spectra of $\gamma$ solanine," Spectrochimica Acta Part A, vol. 66, no. 1, pp. 208211, 2007.

[20] A. Favila, M. Gallo, and D. Glossman-Mitnik, "CHIH-DFT determination of the molecular structure infrared spectra, 
UV spectra and chemical reactivity of three antitubercular compounds: rifampicin, isoniazid and pyrazinamide," Journal of Molecular Modeling, vol. 13, no. 4, pp. 505-518, 2007.

[21] W. J. Hehre, L. Radom, P. V. Schleyer, and J. Pople, Ab Initio Molecular Orbital Theory, Wiley-Interscience, New York, NY, USA, 1986.

[22] J. B. Foresmand and A. Frisch, Exploring Chemistry with Electronic Structure Methods, chapter 4, Gaussian, Inc., Wallingford, Conn, USA, 2nd edition, 1996.

[23] P. R. Westmoreland, P. A. Kollman, A. M. Chaka, et al., Eds., Applications of Molecular and Materials Modeliing, Springer, Berlin, Germany, 2002.

[24] A. Hinchliffe, Ed., Chemical Modelling: Applications and Theory, vol. 5, The Royal Society of Chemistry, London, UK, 2008.

[25] D. Young, Computational Chemistry: A Practical Guide for Applying Techniques to Reald-World Problems, John Wiley \& Sons, New York, NY, USA, 2001.

[26] S. M. Bachrach, Computational Organic Chemistry, John Wiley \& Sons, New York, NY, USA, 2007.

[27] C. J. Cramer, Essentials of Computational Chemistry: Theories and Models, John Wiley \& Sons, Chichester, UK, 2002.

[28] J. M. Seminario and P. Politzer, Eds., Modern Density Functional Theory: A Tool for Chemistry, Elsevier, Amsterdam, The Netherlands, 1995.

[29] A. Hinchliffe, Molecular Modelling for Beginners, John Wiley \& Sons, New York, NY, USA, 2nd edition, 2008.

[30] C. E. Dykstra, G. Frenking, K. S. Kim, and G. E. Scuseria, Theory and Applications of Computational Chemistry: The First Forty Years, Elsevier, Amsterdam, The Netherlands, 2005.

[31] J. C. Slater, Quantum Theory of Molecular and Solids, Vol. 4: The Self-Consistent Field for Molecular and Solids, McGrawHill, New York, NY, USA, 1974.

[32] S. H. Vosko, L. Wilk, and M. Nusair, "Accurate spin-dependent electron liquid correlation energies for local spin density calculations: a critical analysis," Canadian Journal of Physics, vol. 58, no. 8, pp. 1200-1211, 1980.

[33] A. D. Becke, "Density-functional exchange-energy approximation with correct asymptotic behavior," Physical Review A, vol. 38, no. 6, pp. 3098-3100, 1988.

[34] C. Lee, W. Yang, and R. G. Parr, "Development of the ColleSalvetti correlation-energy formula into a functional of the electron density," Physical Review B, vol. 37, no. 2, pp. 785$789,1988$.

[35] A. D. Becke, "Density-functional thermochemistry-part III: the role of exact exchange," The Journal of Chemical Physics, vol. 98, no. 7, pp. 5648-5652, 1993.

[36] J. P. Perdew, J. A. Chevary, S. H. Vosko, K. A. Jackson, M. R. Pederson, D. J. Singh, and C. Fiolhais, "Atoms, molecules, solids, and surfaces: applications of the generalized gradient approximation for exchange and correlation," Physical Review $B$, vol. 46, no. 11, pp. 6671-6687, 1992.

[37] J. Tao, J. P. Perdew, V. N. Staroverov, and G. E. Scuseria, "Climbing the density functional ladder: nonempirical metageneralized gradient approximation designed for molecules and solids," Physical Review Letters, vol. 91, no. 14, Article ID 146401, 4 pages, 2003.

[38] V. N. Staroverov, G. E. Scuseria, J. Tao, and J. P. Perdew, "Comparative assessment of a new nonempirical density functional: molecules and hydrogen-bonded complexes," Journal of Chemical Physics, vol. 119, no. 23, pp. 12129-12137, 2003.

[39] E. Lewars, Computational Chemistry: Introduction to the Theory and Applications of Molecular and Quantum Mechanics, Kluwer Academic Publishers, Norwell, Mass, USA, 2003.
[40] F. Jensen, Introduction to Computational Chemistry, John Wiley \& Sons, Chichester, UK, 2007.

[41] R. E. Stratmann, G. E. Scuseria, and M. J. Frisch, "An efficient implementation of time-dependent density-functional theory for the calculation of excitation energies of large molecules," Journal of Chemical Physics, vol. 109, no. 19, pp. 8218-8224, 1998.

[42] R. Bauernschmitt and R. Ahlrichs, "Treatment of electronic excitations within the adiabatic approximation of time dependent density functional theory," Chemical Physics Letters, vol. 256, no. 4-5, pp. 454-464, 1996.

[43] M. E. Casida, C. Jamorski, K. C. Casida, and D. R. Salahub, "Molecular excitation energies to high-lying bound states from time-dependent density-functional response theory: characterization and correction of the time-dependent local density approximation ionization threshold," Journal of Chemical Physics, vol. 108, no. 11, pp. 4439-4449, 1998.

[44] S. I. Gorelsky, "SWizard program," http://www.sg-chem.net/.

[45] E. E. Castellano, O. E. Piro, J. A. Caram, et al., "Crystallographic study and molecular orbital calculations of thiadiazole derivatives. 2. 3,4-diphenyl-1,2,5-thiadiazole 1-monoxide," Journal of Molecular Structure, vol. 604, no. 2-3, pp. 195-203, 2002.

[46] S. I. Gorelsky, “AOMix program,” http://www.sg-chem.net/. 


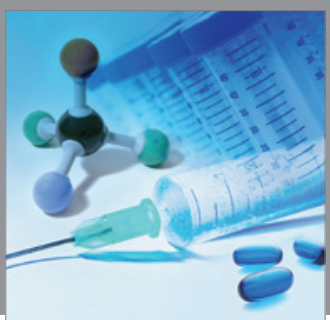

International Journal of

Medicinal Chemistry

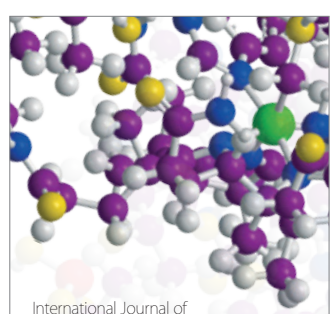

Carbohydrate Chemistry

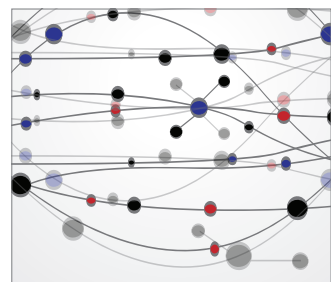

The Scientific World Journal
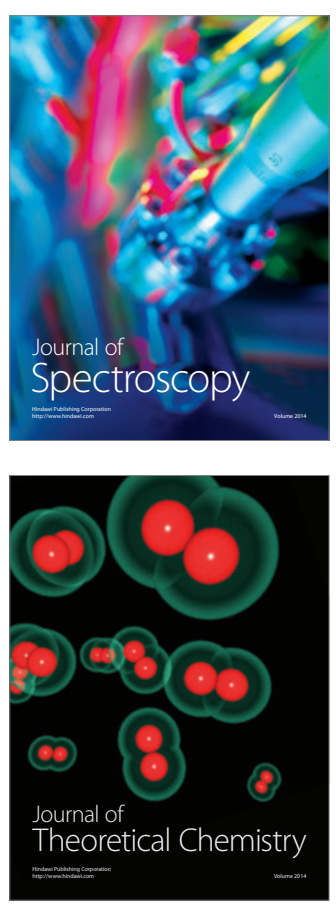
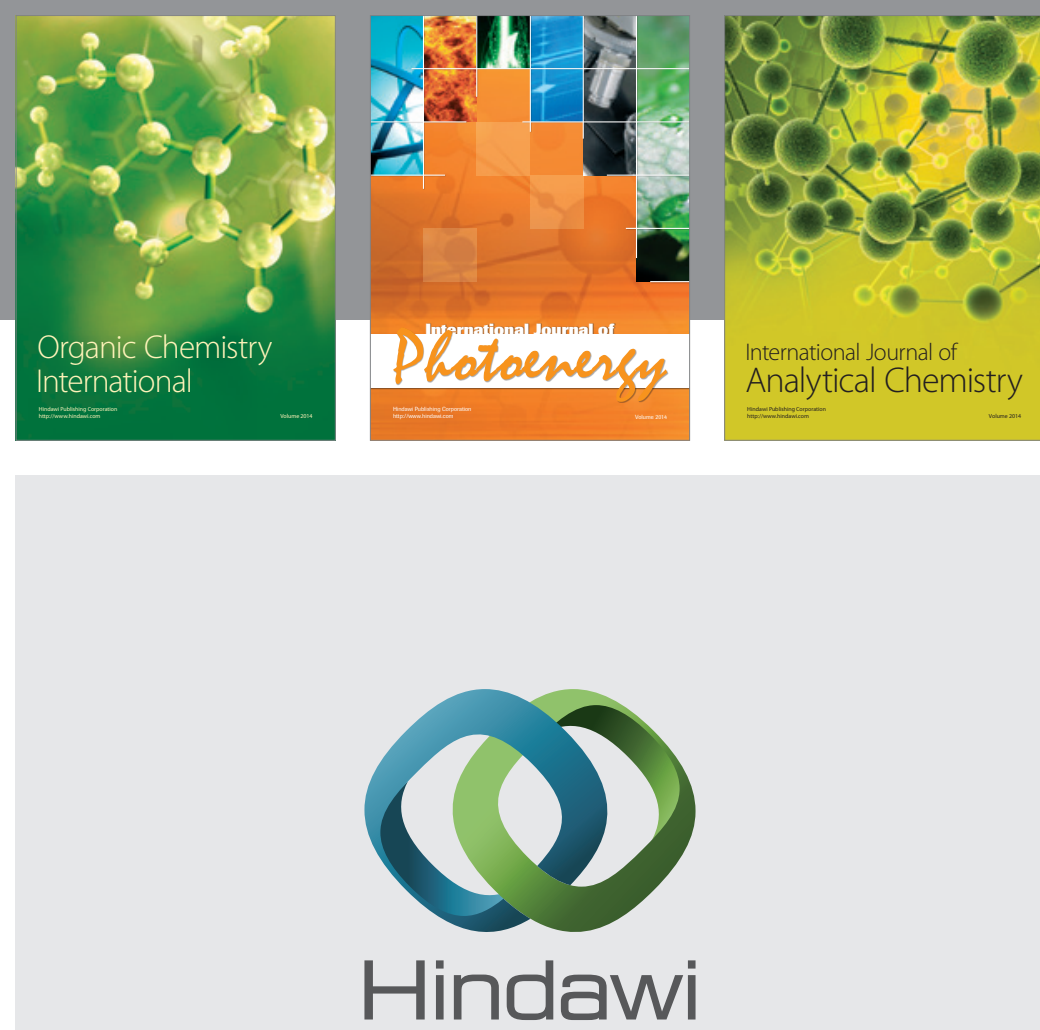

Submit your manuscripts at

http://www.hindawi.com
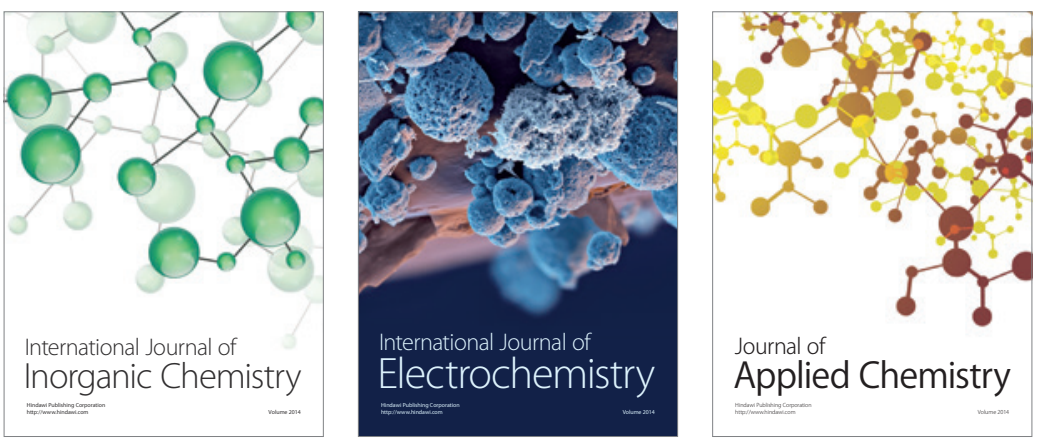

Journal of

Applied Chemistry
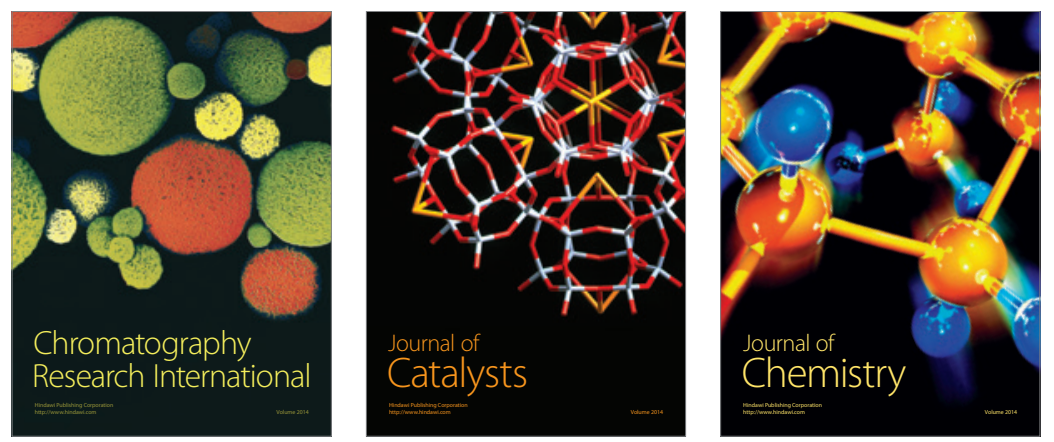
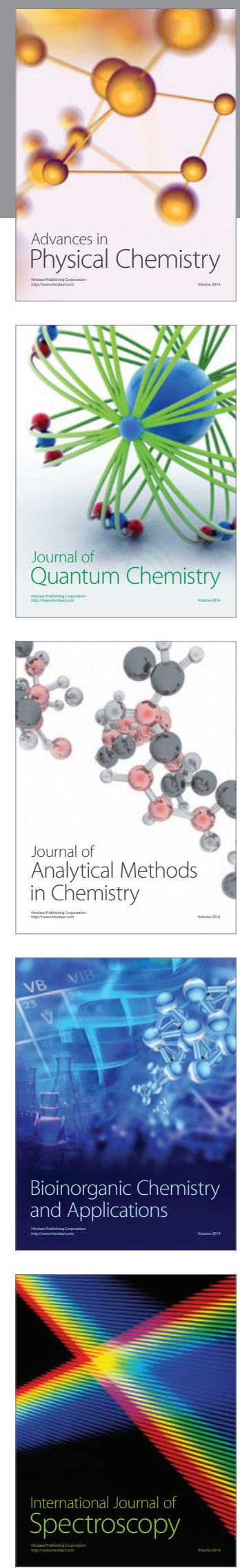